\title{
Understanding User Uncertainty during the Implementation of Self-Service Business Intelligence: A Thematic Analysis
}

\author{
Severin Weiler \\ LMU Munich \\ weiler@bwl.lmu.de
}

\author{
Christian Matt \\ University of Bern \\ christian.matt@iwi.unibe.ch
}

\author{
Thomas Hess \\ LMU Munich \\ hess@bwl.lmu.de
}

\begin{abstract}
Owing to Self-Service Business Intelligence (SSBI) systems' transformative power for organizations, substantial user uncertainties often blight their potential. Although these uncertainties pose a significant threat to effective SSBI implementation, their sources and determinants remain unclear. We conducted semi-structured interviews with 15 current users of a recently implemented SSBI system to empirically explore the relevant factors of user uncertainty. We undertook a rigorous thematic analysis of the collected data, thereafter developing a thematic map to visualize user uncertainties. This map uncovered three unexplored important factors (work routine change, social dynamics and fear of AI) for future research. Our findings show that users are not only perturbed by "hard" factors (e.g. a lack of technical understanding), but also by "soft" factors (social dynamics, fear of AI and nontransparency). Practitioners can use the thematic map to identify and observe potential uncertainties and to develop adequate procedures.
\end{abstract}

\section{Introduction}

Traditionally, decision-makers needed to ask BI specialists, who were either IT specialists or very experienced BI users [1], for Business Intelligence (BI) reports or analyses. The growth of accessible data and the increased application of BI in operative domains have created a demand for new, more flexible and more usable BI systems [2]. In essence, SSBI systems are a novel response to this demand [1, 3]. These systems have been designed to allow users to easily access data without the help of others [3]. SSBI allows experienced BI users (i.e. power users) to perform their tasks quicker and easier, while allowing unexperienced BI users (i.e. casual users) to be more self-reliant [1]. Consequently, SSBI benefits the organization by contributing to a greater operational efficiency [4]. Despite these advantages, SSBI's effectiveness is still low: $64 \%$ of BI professionals rate their success with SSBI as "average or lower" [5]. In addition, the implementation rate has stagnated at 55\% since 2014, despite $15 \%$ of organizations annually stating that they will implement self-service BI during the next 12 months [6].

This sluggish diffusion indicates that managing the SSBI implementation process not only faces technical challenges, but also implementation challenges from the potential users [4]. During the implementation phase, when the usage patterns are unstable and there is little experience with the new system, an SSBI system can specifically perturb users, provoking uncertainties, which, in turn, may hinder them from tapping into SSBI's full potential [7]. Specifically, SSBI's universal applicability and its fundamental influence on existing work routines prevent users from using it effectively. Power users are particularly affected, due to SSBI automating many aspects of their daily work. However, prior research into SSBI implementation challenges has primarily focused on the organizational perspective [4]. No study has as yet offered empirical evidence of the main determinants of user uncertainty during SSBI implementation. In addition, no theoretical approaches have been suggested to predict users' behavior towards SSBI in the implementation phase. We therefore introduce the uncertainty concept into the SSBI discourse, arguing that unveiling SSBI's specific sources of user uncertainty is the basis for identifying ways to reduce it [7]. In turn, these ways of reducing uncertainty could inspire the development of prescriptive models [e.g. 7, 8] that provide the information required to better explain user uncertainties or usage inefficiencies during the SSBI implementation. We therefore intend to answer the following research question empirically: What are the determinants of user uncertainty during the implementation of SSBI?

To answer this research question, we adopted Venkatesh et al.'s [7] theoretical perspective of user 
uncertainty during the implementation of technology, who distinguish between three types of uncertainty (i.e. task uncertainty, workflow uncertainty, and environmental uncertainty). Given the scarce amount of literature in this field, we used a qualitative approach and conducted 15 semi-structured interviews with users of a recently introduced SSBI system in the context of supply chain management. Subsequently, we undertook a rigorous, iterative thematic analysis to empirically unveil and map the factors related to three theory-grounded dimensions of uncertainty. The research call for an "[...] in-depth knowledge regarding how organizations interpret the identified [SSBI implementation] challenges" and for research that "[...] could validate or extend the identified challenges" motivated our study [4, p. 5062]. The thematic map addresses this call by providing researchers with a better understanding of the sources (i.e. factors) of user uncertainties during the implementation of an SSBI. Furthermore, this thematic map helps practitioners manage uncertainties in an SSBI implementation process.

\section{Theoretical background}

\subsection{SSBI systems}

BI systems' goal is to enable organizations to apply data-based decision-making efficiently [1]. Since BI systems' functional scope varies substantially, manifold definitions have been used to describe it [9]. The BI term has been used for simple reporting tools for technical infrastructures, like data warehouse systems, but also for advanced analytical tools, like data mining or predictive analytics [9]. Chen et al., who depict BI (and analytics) as encompassing underlying data processes and analytical technologies, as well as "business-centric practices and methodologies," propose a holistic definition to grasp this diversity conceptually [10, $\mathrm{p}$. $1166]$.

While maintaining BI's general goal, SSBI systems "simplify the process of BI use, therefore enabling power and casual users to make use of BI outputs" [4, p. 5058]. This simplicity can be broken down to an "easier access to source data for reporting and analysis, easier and improved support for data analysis features, faster deployment options such as appliances and cloud computing, and simpler, customizable, and collaborative end-user interfaces" [3, p. 4]. By providing a universal and accessible platform, SSBI provides users with new possibilities to utilize BI. Casual users can now create BI analyses and reports without help. Power users, on the other hand, will experience SSBI transforming their work processes by facilitating them and assuming tasks by automating them [1]. Besides usability aspects, SSBI enables organizations to integrate new types of data sources and to utilize BI systems in operational domains [1]. SSBI therefore represents a significant shift in user interaction through the fundamental way it differs from traditional BI in terms of user behavior and user groups.

Notwithstanding SSBI's benefits, a number of organizations have struggled with implementing this technology [4]. During the implementation phase, SSBI's general advantages (its universal applicability and the transformative power it has in respect of users' daily work) turn into implementation barriers by making users uncertain. To ensure SSBI's effective use, organizations need to address these barriers during the implementation phase. IS researchers have contributed to a rich body of knowledge on the usage of BI at the individual level and the factors or barriers contributing to a seamless or restricted usage process. This research covers both the organizational perspective [e.g. 11] and the individual perspective [e.g. 12]. Despite these contributions, the transfer of findings from the traditional BI systems context to the SSBI context is restricted, due to the latter systems differing fundamentally from the former systems in terms of applicability and in respect of users' daily work.

\subsection{User uncertainties}

Economists view uncertainty as resulting from a lack of information about the future in a decisionmaking situation [13]. The uncertainty concept found broad application in the context of organizational behavior [e.g. 14-16] and consumer behavior [e.g. 8, $17,18]$, thereby addressing career uncertainty [16], environmental uncertainty [15], fairness uncertainty [14], agency uncertainty [8], and product uncertainty $[17,18]$. In the technology context, uncertainty is an individual's perception of being unable to predict or understand the technology environment [19].

Individual users' perceptions of and behaviors towards technology can be structured into four sequential individual-focused variables: acceptance, intention to use, use, and user satisfaction [20]. Uncertainty occurs especially during this model's first three phases, due to the user's lack of prior experiences with the system [7]. This is particularly true during the implementation of a new software within an organization. Throughout the study, we therefore mention user behavior, which refers to the depicted model's first three variables, during the implementation of an SSBI system. 
Studies from the field of IS research applying the uncertainty concept to questions related to an individual's usage of technology during its implementation are rare. The study by Venkatesh et al. [7] on users' behavior during the implementation of an e-government service is an exception. These authors posit that there are three dimensions of uncertainty during the implementation of a new technology: (1) task uncertainty, (2) workflow uncertainty, and (3) environmental uncertainty. Figure 1 schematically illustrates the three dimensions of user uncertainty, which we will outline in the following. In Figure 1, $t$ represents the time dimension of a user's interaction process with the SSBI.

Task uncertainty is "the difference between the amount of information required to perform the task and the amount of information already possessed" [21, pp. 36-37]. In the context of an SSBI's implementation, this means that the users need to have the knowledge required to use the system in the way it is intended to be used. In respect of SSBI systems, task uncertainty can materialize in the form of incorrect queries/inputs, missing user instructions on how to select, interpret, and analyze data that the SSBI provides, but also as a lack of understanding of the SSBI system's technical peculiarities (e.g. product increments).

In its original context, i.e. the management of the production of goods, "workflow uncertainty refers to knowledge about when the inputs will arrive at an individual's station to be processed" [22, p. 195]. In the context of implementing an SSBI, workflow uncertainty describes information gaps about how and when the interaction with the new system will take place, resulting in an ineffective interaction process [7]. In respect of SSBI systems, this refers to a lack of information about how and when the user should initiate, pause or resume her usage of the system, particularly after irregularities (e.g. errors) in the usage.

Environmental uncertainty is "the extent to which critical information about organizations, activities, and events is unknown. It also pertains to lacking clarity about cause-and-effect relationships among environmental elements" [20, p. 188]. The more unstable, complex, and dispersed the environment, the higher the level of environmental uncertainty [23]. In new technologies, this environmental uncertainty mainly stems from the influence that unpredictable environmental factors have on the user's experience. In SSBI, these factors may include the social dynamics within the organization, individual user preferences or performance risks associated with the functionalities that the SSBI offers.

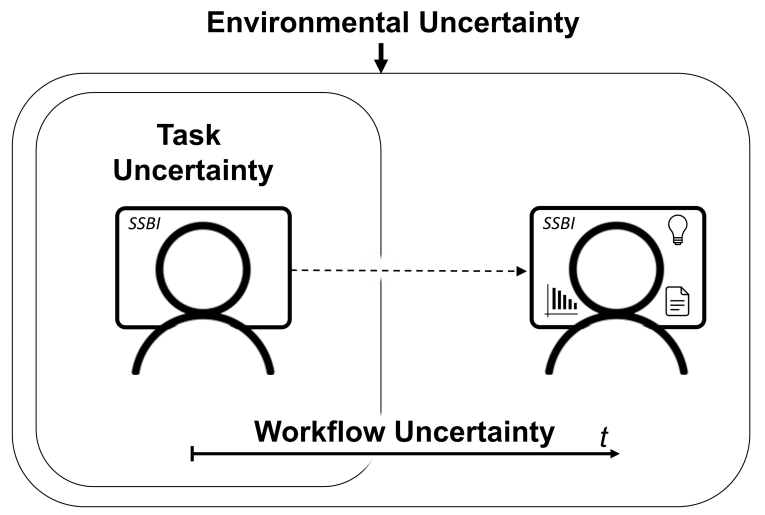

Figure 1. Dimensions of user uncertainty

\section{Data collection and analysis}

We used a rigorous qualitative research approach for the literature review, to conduct interviews, and, ultimately, to analyze the data [24-26]. A qualitative research approach is particularly suitable to answer our explorative IS research question [24], which aims to answer questions such as: "what is happening?" and "why is it happening?" $[24$, p. 6]. To obtain a detailed understanding of these questions, we conducted 15 semi-structured expert interviews [26].

The data were collected in cooperation with a multinational IT company that introduced an SSBI during the first half of 2017. At the time of the analysis (Spring 2018), the company's SSBI provided features ranging from access to information, drilling anywhere, to the automated creation of reports and advanced analytics. It could be classified as an SSBI with low to medium self-reliance and low to medium system support [1]. In addition to a menu-based navigation, the SSBI could be used by asking questions in written natural language. These questions were drawn from a list that a support and development unit updated constantly. Future versions of the SSBI will provide decision support and undertake specific work processes autonomously.

Since we were interested in the users' experiences during the implementation of an SSBI system, we only interviewed those already using the SSBI. Furthermore, we focused on the experiences of power users, who quantitatively represented the biggest user group within the case company and whose daily work changed most during the implementation process. Consequently, they represent most of our sample. We also interviewed a smaller number of casual users to gain a comprehensive understanding of the SSBI and its organizational context. When we conducted the interviews, the SSBI's usage was for all means and 
purposes nonmandatory, although the management did suggest they do so. Since the SSBI's implementation process was tailored to the needs of a specific subdivision, thus differing slightly across subdivisions, we focused on just one subdivision (Supply Chain Assurance) to ensure that the implementation process was similar for all the interviewees.

In the selection of interview partners, we relied on the purposive sampling technique, thus ensuring that we covered all the relevant user groups within the organization [27]. Following the recommendations by Marshall et al., we collected data until we achieved data saturation [28]. In total, we conducted 15 semi-structured interviews (face-to-face: 1; telephone: 1; video chat: 13) with experts who had experienced the SSBI's implementation as power or casual users. The interviews were conducted in English. Six of the subjects are female, nine male, and they are all on average 42.7 years old. Eight of the interviewees have a master degree, seven a bachelor degree. Ten of the 15 can be depicted as power users, whereas the other five are casual users. Our interview guideline consisted of 31 open-ended questions, with an interview lasting between 44 and 85 minutes. The interview guideline consisted of three parts, namely the (1) experts' daily work and their use of software, (2) their experiences during the implementation of the SSBI, and (3) their opinion of the system's development and the future challenges. We recorded, transcribed, and analyzed the interviews according to qualitative data coding standards [24]. The coding and analyzing were done with Atlas.ti, one of most widely used software for qualitative data analysis by IS researchers [29, e.g. 30]. We chose the thematic analysis method to evaluate the collected interview data [25]. The thematic analysis approach allows the researcher to descriptively and interpretatively reveal themes (i.e. patterns) within the collected data, which can then be used to create maps or tables of analysis. This analysis is particularly suitable for sensitive data environments [27] and is frequently employed in IS research [29, e.g. 30]. We followed the iterative approach, which is based on theory, to the thematic analysis [30], thus following a constructionist epistemology [31]. This means we already had a specific research question in mind while looking for latent (i.e. underlying) and manifest (i.e. apparent) themes that could foster our understanding of the reported information and its socio-cultural context. An iterative thematic analysis approach follows four steps. The first is to transcribe the interviews verbatim and to read through them repeatedly. Second, we searched for patterns and coded these. In a third step, these codes were merged into thematically coherent themes. Subsequently, the themes were matched with the three theory-grounded dimensions of uncertainty during the implementation of technologies (i.e. task, workflow, and environmental uncertainty). The second and third steps were taken while constantly reviewing the literature.

\section{Results}

The thematic map (Figure 2) visualizes the results and comprises the three theory-grounded dimensions of uncertainty that we explained in Section 2. The thematic map is used to allocate the eight factors of uncertainty identified in the data to our framework. These eight data-based factors will be described in the following sections.

\subsection{Task uncertainty}

We related three factors to the task uncertainty dimension: Incorrect use, lack of technical understanding, and performance mistrust.

Incorrect use: Users struggle to use the SSBI system the way it is intended to be used: "I cannot use the system as I would like to use it" [P2]. Despite being provided with a list of specific queries for the SSBI system, the users used queries not on the list: "We said, 'here's a list of the questions the SSBI recognizes.' But they typed in what they wanted" [P3]. In the same vein, users transferred usage pattern from their private life to the new SSBI: "People started using the SSBI as if it were Alexa" [P3]. Owing to its complexity and it being newly implemented, there are no standardized training programs or comparable training environments for the SSBI system: "So there's little technology on which to train them [users] on how to operate the interface" [P4]. Not only does their lack of knowledge perturb the users, but also the lack of training programs, which used to be the rule when software tools were previously introduced.

Lack of technical understanding: A technical understanding of the SSBI system was said to have a major influence regarding reducing user uncertainty. The relationship between the understanding of technology and user uncertainty stems specifically from components that are, from the user perspective, in the system's background: "I know what's behind it, but not everyone else knows what's behind it [...]. So, I'm not skeptical of the tool, but other people are" [P4]. With the SSBI in place, algorithms and data structures are specifically the technical components that make users feel uncertain. The users had 
previously experienced the functionalities of the SSBI and its system architecture in other software, which meant they were less unsure about these technical aspects. The functionalities represent the benefit that users gain from the SSBI system, while the system architecture refers to the SSBI as a comprehensive system built on the established data warehouse systems with which the users had previously worked. On average users who were involved in the development process (e.g. participating in focus groups on product requirements, beta-phase feedback, etc.) showed a better technical understanding of the SSBI in contrast with those not involved in the creation process, for example, "It's not like the machine does it by itself, but the user can feedback the system with the interface" [P6]. Consequently, they had a better understanding on how a SSBI system is developed.

Performance mistrust: The SSBI under investigation had data integrity flaws that mainly originated from the SSBI-specific goal of being a meta access point for various databases: "At the beginning, the information that the SSBI was displaying was not in concordance with other systems" [P6]. Users perceived this as an indicator of the software's immaturity, which perturbed them: "[...] if you know that the system will anyhow change and new releases come regularly, you feel that it's not ready yet" [P8]. Furthermore, there were different understandings of what the users could expect from the software. Some users, for example, showed little tolerance of the SSBI's mistakes: "During the fifth phase [i.e. software updates], it [data error] was justified. But now, we shouldn't have any discrepancies" [P6]. Negative experiences during the SSBI implementation phase resulted in strong skepticism about the system's performance: "I think I've been resistant to it just because it hasn't been hundred percent capable yet" [P5]. This mistrust is also highlighted when examining the work practices of users who built their own workarounds to cope with the SSBI's imperfection: "I find an answer on the SSBI and then I go double check it and one of my traditional tools just to make sure that it's on the same page." [P5]. The first experiences with the new SSBI were especially critical for the users' perception of the SSBI's usefulness and influenced their attitude towards the implementation significantly: "What I can see from other people is that they expect immediately that everything should be perfect. They are really unhappy if something is not perfect and then they say that it is not usable" [P2]. Generally, we found that the users had very high expectations of the performance of the SSBI: "That's that we think that human can make mistakes, but machines can' $t$ "'
[P7]. This misguided expectation perturbed users' when using the SSBI system.

\subsection{Workflow uncertainty}

We identified three factors that related to workflow uncertainty: work routine change, intraorganizational standards, and social dynamics.

Work routine change: Generally, we found that "people are scared of change. They're scared of how that change can impact their daily work" [P5]. This fear of change can be linked to a specific SSBI aspect: its comprehensiveness. Users were skeptical about the SSBI's future importance within their organization, because its complexity threatens its success within the organization. In addition, the SSBI has the potential to impact many tasks within the investigated departments. These two aspects mean the SSBI could do far more harm than previously introduced systems whose failure the users had experienced: "They want to wait and see if it can live first because why waste your time if it is just gonna go away in a year like other systems" [P3]. Before committing to a new system and integrating it into their existing work routines, the users need to know that the SSBI system's implementation is sustainable: "First time out [release of the system] was the hardest. Their [users] trust wasn't there, their interest wasn't there, because they didn't believe that this was going to last" [P3].

Intraorganizational standards: Owing to the multinational corporate structure, many of the business processes and terminologies being used differ across the organization's subdivisions. However, all of the organization's subdivisions and departments should be able to use the SSBI to guarantee the data's consistency and optimal efficiency gains. When we collected the data, most of the processes and terminologies that the SSBI applied were not standardized: "It turned out that we are using some different meanings behind the terms. [...] the three departments were not on the same track. Everybody used the system in a different way, according to their needs and parameters." [P2]. This lack of business process and terminology standardization across the organizational functions and departments led to unexplainable errors and futile interaction processes: "When you involve different manufacturing sites with different products and processes, you have to be very, very clear on the basic terminology [...]. [...] and then you realize, while you are using the SSBI, that something is not going well" [P8].

Social dynamics: The organizational structure of the organization emphasizes the role of teams. Users 
are used to working in teams and having co-workers around them with similar tasks, making their workrelated experiences comparable: "People work more as a team and there is a lot of influence between peers" [P9]. Consequently, the team's usage patterns influenced the software usage decisions: "And people do react to seeing someone who is recognized or being a user of this new AI tool. [...] So there is a lot of peer pressure in the whole system. So, not so much of individualism" [P9]. The SSBI has, to a certain extent, transformed this team structure, by shifting some of the work responsibility from the team to individuals. The SSBI allocates more responsibilities to the user, which will shift various departments' tasks from teams to individuals. This can be a challenge for SSBI's implementation, as users covertly resist using it. This resistance stems from the established social groups' drive for self-preservation, which materializes in reactionary user activity aimed at maintaining the status quo (pre-SSBI) regarding social practices: "[...] he felt more comfortable to call me from Mexico in the evening (my time), instead of looking at the order status in the SSBI' [P8]. Users reported strongly feeling part of a team and viewing their work tasks as a team effort. However, with the help of the SSBI, casual users can perform many tasks themselves, no longer needing to rely on power users' assistance.

\subsection{Environmental uncertainty}

We identified two factors of the environmental uncertainty dimension: fear of AI and non-transparency.

Fear of AI: Users reported fear of the SSBI related to it being associated with artificial intelligence (AI) technology. Although, technologywise, the SSBI might not yet meet the definition of an intelligent system, both its given name and the available descriptions (e.g. "cognitive tool") suggest that it is: "When I heard about it, I thought of artificial intelligence" [P7]. This triggers associations that users perceive as threatening. First, users feared that the SSBI would soon replace their job: "I also have kind of a fear. [...]. Right now I'm working on a system that will be able to answer the questions that were answered by me in the last years. And what I will do in 10 years?" [P2]. The users believed that the SSBI could automate their current tasks: "[I]t's [the SSBI] kind of replacing a lot of the analysis that me and my team are doing now. People see machines becoming more and more autonomous and being able to do more and more jobs. I think people are scared about their own personal jobs." [P6]; "My fear is, it will do a lot of things instead of us in 10 years, which doesn't make me happy" [P8]. Second, users were perturbed about using SSBI, due to their privacy concerns. They believed that they were contributing to the development of technology threatening privacy through their usage data: "And I think also because in any job people are afraid of an AI. They think that it's like spying on him maybe or its learning from what you do" [P4]. Since the data disclosure occurs in a professional context, the users perceive their situation as paradoxical: "[R]ight now I'm teaching something which will take my job in the future" [P2]. The more the users work with the SSBI (i.e. the more user data they provide), the more they contribute to the development of a technology that might, in a later version, automate some of their tasks.

Nontransparency: The SSBI users are perturbed due to the opacity of the systems' functionalities and of the organizational strategy behind the SSBI's implementation. During the implementation phase, when usage experience is scarce, users specifically require the system to provide technical explanations that the SSBIs information can be trusted: "[T]hey aren't trusting a black box. They say: 'Show me how you got there'. And the SSBI would then have to show all the pieces of information" [P3]. These

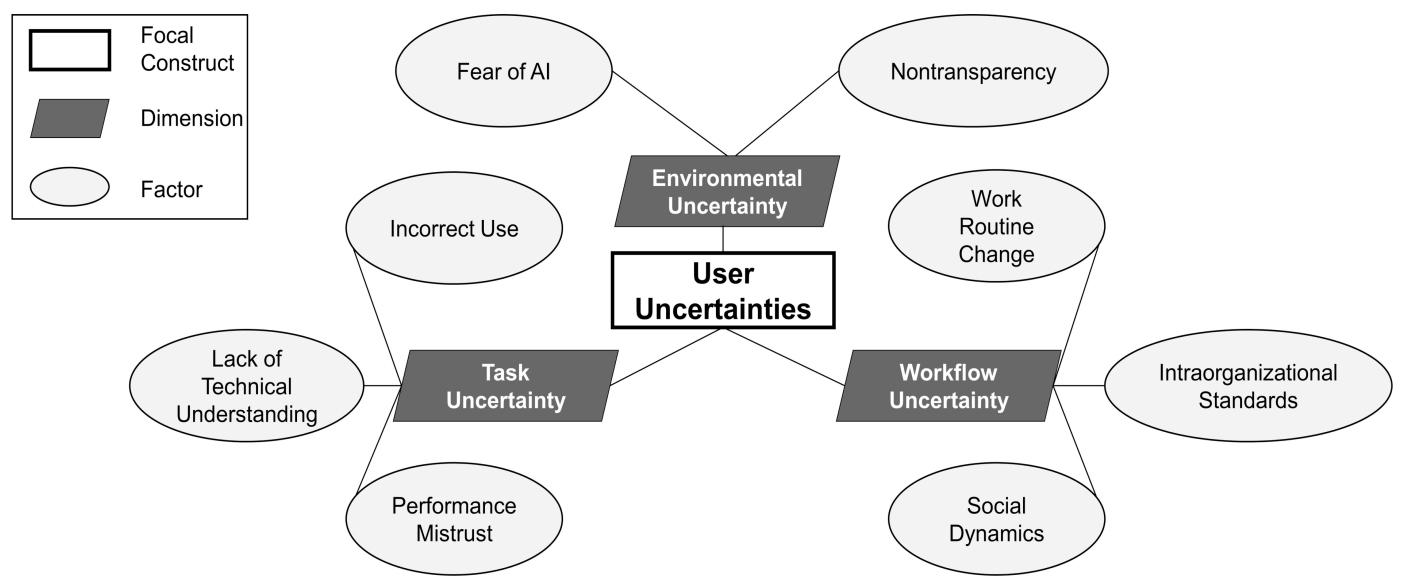

Figure 2. User uncertainties during the implementation of SSBI 
explanations should unveil the sources of the used data and the analysis that the SSBI applied: "[N]ot just providing an answer, but having explanations and being able to walk you through how it got to an answer" [P4]. In addition to the technical opacity, the lack of clarity about the organization's strategy regarding the SSBI's implementation makes the users uncertain: "I tell you the truth I don't understand why the company is calling itself cognitive" [P10]. The users were in fact told that the new SSBI is part of the "cognitive company" and that this approach would transform many aspects of the organization's traditional business, including its work organization and value chains, making the organization more efficient. First, users find the way these efficiency gains will be distributed very opaque: "You know, the big questions is what will the company do with $40 \%$ of employees' time savings. So, cancel the jobs or educate all that people to use [company's name] culture in other businesses. That is a big question and that is absolutely a decision on the management level." [P10]. Second, how the organization's strategy will transform the users' daily work is also still opaque. The nontransparency of the organization's strategic objectives perturbs users during their first encounter with the strategy's operational objectives (i.e. the SSBI).

\section{Discussion}

In the following, we discuss the three factors (i.e. work routine change, social dynamics, and fear of AI) that the literature on challenges during an SSBI implementation has not yet covered [4].

Work routine change: The SSBI users were skeptical about its sustainability and thus hesitant to integrate it into their established work routines. Work routines make use of information, technology, and other resources to produce goods or services [33]. These three components need to be aligned to enable users to perform their work routines effectively [33]. We found that the users lacked this alignment during the SSBI implementation, thus hindering them from using it effectively. Although the material aspect of the work routine changed as a result of the SSBI implementation, the users did not have sufficient information and resources to alter their existing work routines or to develop new ones. The users reported that they were unsure about how to employ the SSBI to make their work more effective, because their tasks remained the same while they could still use the current software. They were mostly unsure about the range of functionalities the SSBI provided and, therefore, only rarely realized how the SSBI could benefit their work. Consequently, we support previous findings on the strong influence that users' perception of their work routines has on their resistance to a new system [34]. We also found that users were particularly skeptical of changing their work routines, due to the high expectations of the SSBI's implementation, which exceeded previous software implementation projects in reach and comprehensiveness. This finding confirms finding in previous work, suggesting that users' resistance to new IS is strong if the system is comprehensive and affects the users' work routines [35].

Social dynamics: We conducted the interviews in a business department characterized by a team-based organization. We found that, according to the users' perception, the SSBI's implementation challenges the team structure, thus perturbing the users in the implementation phase. Working in a team successfully involves users internalizing (i.e. act in accordance with) the group's opinions and identifying with the group (i.e. adopt conformable behaviors) [36]. In the users' perception, the SSBI's implementation challenges their respective social group's team-based orientation. They thus react by covertly resisting the SSBI to maintain their social structure. This resistance is manifested in their uncertainty about using the system. Furthermore, we found that, during the SSBI's implementation, social norms influence the users' behavior. Social norms are the dominant way of conceptualizing social influence in IS research [37] and is defined as the "perceived social pressure to perform or not to perform the behavior" [38]. Social norms have been found to be particularly valuable for predicting initial user behavior [39]. Having gained experience with the system, the users tend to rely less on these social norms. In our study, we support this sequence: Users who had used the SSBI for some time tended to talk less about their fellow employees than those whose user experience was still very scant. The latter referred more often to their colleagues' user experiences and, in general, showed greater aversion to using the SSBI.

Fear of AI: The SSBI is transforming the work processes, which is associated with the users' artificial-intelligence-caused negative emotional responses, which perturb them in the implementation phase. To date, previous works on the resistance and acceptance of technologies have largely disregarded the role of emotional responses [40], because IT theories tend to be based on the assumption of rational-analytic and utility-maximizing agents [41]. By showing the relationship between the implementation of an SSBI system and negative user affects, we support recent findings showing that, as a stimulus, IT can induce emotions, thus shaping 
different forms of user response [42]. In our study, the users associated the SSBI with fear. We found that this emotional reaction mainly stemmed from two aspects. First, the SSBI transforms traditional work processes by enabling casual users to do various tasks without help and helps power users automate many of their tasks. The second aspect is our finding that the users associated the SSBI with AI technologies. The users quickly realized the potential the SSBI had for their daily work. The SSBI optimizes a traditional BI request's time-consuming process, saving the users a great deal of time. However, despite viewing the SSBI as a helpful tool, the users primarily exhibited fearful and intimidated reactions. This negative reaction is related to the context of meaning within which the SSBI operates. The users associated AI technologies with the threat of losing their jobs and with being monitored, especially in the labor context. This perturbed these users and influenced their SSBI usage behavior, as they were very doubtful about the benefits that the SSBI could provide. Such an emotional response could lead them to psychologically distance themselves from the SSBI [42].

\section{Implications}

\subsection{Implications for research}

The study has several important theoretical implications. First, the thematic map provides researchers with a visual structure of the factors determining user uncertainty during an SSBI's implementation. The map can therefore be viewed as a strong initial insight into the main determinants of user uncertainty during an SSBI's implementation. This study's results could contribute to an understanding of user uncertainty in the context and identify avenues for future research. A quantitative research design could, for example, determine the relationship between the observed factors and the thematic map's dimensions.

Furthermore, we extend previous research on the challenges during an SSBI's implementation by identifying three important factors (i.e. work routine change, social dynamics, and fear of AI) that prior research on an SSBI's implementation has not yet discussed [4]. Prior research on SSBI's implementation challenges could be divided into "access and use of data" and "self-reliant users" [4, p. 5057]. We see our findings as contributing to the second category (i.e. self-reliant users). Furthermore, our study's findings support IS research focused on the role of emotions in IT use. By describing the "subjective experience of agency," we shed light on how human agents feel about themselves and their environment when a new technology is implemented in their organization [41, p. 212]. Since most studies on the role of emotions focus on the private context [e.g. 42], we enrich this literature stream by offering an insight into the professional context.

\subsection{Implications for practice}

The thematic map could serve as a practical guideline for managers to develop successful SSBI implementation strategies. While organizations frequently implement new IS, the employed technologies are often not used to their full potential, because employees resist the new IS [43]. Prior research has shown that a reason for the ineffective employment of IS is organizations failing to manage employees' perceptions [44]. The thematic map helps managers identify the sources of uncertainty and will therefore help organizations better manage the uncertainty associated with an SSBI's implementation [7]. We suggest that organizations should strategize the following three aspects when implementing an SSBI.

First, organizations should be careful with branding the SSBI as an intelligent system and strive for transparency regarding the SSBI's process/task level. We found that AI perturbs SSBI users, because they associate it with monitoring and the potential threat of losing their jobs. Managers overseeing an SSBI's implementation should carefully consider the image that they want the system to have within the organization. The benefits of branding the SSBI as an intelligent system, whether technologically justified or not, should be weighed against the impact that user uncertainty about using the system could have.

Second, managers should ensure that users know how to integrate the SSBI into their established work routines, which should make sense for the users, from both an outcome perspective and a process perspective. If users have been insufficiently trained to integrate the SSBI into their routines, they will feel uncertain about whether to use it or not. The same applies when the user can achieve the same outcome with current tools. Organizations should address this by extending the existing training programs with modules that enable the users to show their work routines and identify the potential that SSBI could have for the work.

Third, organizations should be transparent about the impact that SSBI will have on the involved departments' social structure. Managers need to acknowledge that their organization's teams are selfreproducing social micro-systems threatened by software that enables less experienced users to 
perform many activities without help. These concerns need to be proactively managed by solving them strategically or by creating a new social structure that to compensate for the established one's decreasing importance.

\section{Limitations, future research and concluding thoughts}

The small sample size of 15 people who participated in the interviews is one of this study's limitations. However, as we conducted a single-case study, this number of participants is comparable to other qualitative studies in ISR [28]. Furthermore, our results are limited, as our object of investigation only offered low to medium self-reliance and system support [1]. High self-reliance and system support were not yet integrated, which meant that some of the users might have perceived the SSBI system as immature software. This perception was also partly due to the SSBI being branded as AI and marketed (internally) as having a major impact on the organization's IT structure, although it was not at this level technologically. We addressed this issue in the interview by pointing out that we were only interested in the version in place during the SSBI's implementation and asking the interviewees to ensure that their answers only referred to this version. Lastly, our study is limited, because we don't know how strongly the identified user uncertainty factors influence the users' long-term resistance or usage of a new SSBI.

Future research should build on our findings, which extend the common body of knowledge in this field, by integrating them into prescriptive models. We specifically deem our findings on user uncertainties during an SSBI's implementation as enhancing existing prescriptive models of user adoption or resistance behavior. Second, our findings on the role of environmental uncertainty hint at different affective cues occurring during an SSBI's implementation. Future research should aim at exploring the role that these affective cues have on users' behavior and perception during an SSBI's implementation. Third, we found that social influence (i.e. identification and internalization) is an important source of user uncertainty; future research should therefore investigate the circumstances under which these mechanisms challenge an SSBI's implementation.

To conclude, our study examined user uncertainty during an SSBI system's implementation. Our qualitative research allowed us to uncover uncertainty factors that users perceive during such an implementation and which may influence their decision to use or resist the system. To achieve this, we conducted 15 expert interviews with users of a recently implemented SSBI system and evaluated them following an iterative thematic analysis. Our findings have shown that users are not only perturbed by "hard" factors (e.g. a lack of technical understanding and work routine change), but also by "soft" factors (social dynamics, fear of AI and nontransparency). These soft factors need to be addressed to successfully implement an SSBI in an organization, specifically because an SSBI system attempts to transform work processes across an entire organization. We hope that our study will spark future research interest, leading to the investigation of the identified uncertainty factors and going beyond the traditional perspectives on the challenges during an SSBI's implementation.

\section{References}

[1] Alpar, P. and Schulz, M., "Self-service business intelligence", Business \& Information Systems Engineering, 58(2), 2016, pp. 151-155.

[2] Yu, E., Lapouchnian, A., and Deng, S., "Adapting to uncertain and evolving enterprise requirements - the case of business-driven business intelligence", in Proceedings, IEEE Seventh International Conference on Research Challenges in Information Science, Paris, France. 2013.

[3] Imhoff, C. and White, C., "Self-service business intelligence", Empowering Users to Generate Insights, TDWI Best practices report, TWDI, Renton, WA, 2011.

[4] Lennerholt, C., van Laere, J., and Söderström, E., "Implementation Challenges of Self Service Business Intelligence: A Literature Review", in Proceedings, 51st Hawaii International Conference on System Sciences (HICSS), Big Island, USA. 2018.

[5] Eckerson, W., "Business-driven BI: Using New Technologies to Foster Self-Service Access to Insights". https:/www.tableau.com/learn/whitepapers/businessdriven-bi, accessed 5-29-2018.

[6] BARC, "Top Business Intelligence Trends 2017". https://bi-survey.com/top-business-intelligence-trends2017, accessed 5-29-2018.

[7] Venkatesh, V., Thong, J.Y.L., Chan, F.K.Y., and Hu, P.J.H., "Managing Citizens' Uncertainty in E-Government Services: The Mediating and Moderating Roles of Transparency and Trust", Information Systems Research, 27(1), 2016, pp. 87-111.

[8] Pavlou, P.A., Liang, H., and Xue, Y., "Understanding and mitigating uncertainty in online exchange relationships: A principal-agent perspective", MIS Quarterly, 31(1), 2007, pp. 105-136.

[9] Marjanovic, O. and Dinter, B., "25+ Years of Business Intelligence and Analytics Minitrack at HICSS: A Text Mining Analysis", in Proceedings, 50th Hawaii International Conference on System Sciences (HICSS), Manoa, USA. 2017. 
[10] Chen, H., Chiang, R.H.L., and Storey, V.C., "Business intelligence and analytics: From big data to big impact", MIS Quarterly, 36(4), 2012, pp. 1165-1188.

[11] Malladi, S., "Adoption of Business Intelligence \& Analytics in Organizations-An Empirical Study of Antecedents", in Proceedings, 19th Americas Conference on Information Systems (AMCIS), Chicago, IL. 2013.

[12] Shibl, R., Lawley, M., and Debuse, J., "Factors influencing decision support system acceptance", Decision Support Systems, 54(2), 2013, pp. 953-961.

[13] Knight, F., Risk, uncertainty and profit, HardPress Publishing, Miami, FL, 2013.

[14] Lind, E.A. and van den Bos, K., "When fairness works: Toward a general theory of uncertainty management", Research in Organizational Behavior, 24, 2002, pp. 181-223.

[15] Sia, C.-L., Teo, H.-H., Tan, B.C.Y., and Wei, K.-K., "Effects of Environmental Uncertainty on Organizational Intention to Adopt Distributed Work Arrangements", IEEE Transactions on Engineering Management, 51(3), 2004, pp. 253-267.

[16] Brashers, D.E., "Communication and Uncertainty Management", Journal of Communication, 51(3), 2001, pp. 477-497.

[17] Matt, C. and Hess, T., "Product fit uncertainty and its effects on vendor choice: An experimental study", Electronic Markets, 26(1), 2016, pp. 83-93.

[18] Daugherty, T., Li, H., and Biocca, F., "Consumer learning and the effects of virtual experience relative to indirect and direct product experience", Psychology and Marketing, 25(7), 2008, pp. 568-586.

[19] Song, M. and Montoya-Weiss, M.M., "The Effect of Perceived Technological Uncertainty on Japanese New Product Development", Academy of Management Journal, 44(1), 2001, pp. 61-80.

[20] Larsen, K.R.T., "A taxonomy of antecedents of information systems success: Variable analysis studies", Journal of Management Information Systems, 20(2), 2003, pp. 169-246.

[21] Galbraith, J., Designing complex organisations, Addison-Wesley, Reading, MA, 1973.

[22] Slocum Jr, J.W. and Sims Jr, H.P., "A typology for integrating technology, organization, and job design", Human Relations, 33(3), 1980, pp. 193-212.

[23] Aldrich, H.E., Organizations and environments, Prentice-Hall, Englewood Cliffs NJ, 1979.

[24] Myers, M.D., Qualitative research in business \& management, SAGE Publications, London, 2009.

[25] Braun, V. and Clarke, V., "Using thematic analysis in psychology", Qualitative research in psychology, 3(2), 2006, pp. 77-101.

[26] Myers, M.D. and Newman, M., "The qualitative interview in IS research: Examining the craft", Information and Organization, 17(1), 2007, pp. 2-26.

[27] Patton, M.Q., Qualitative evaluation and research methods, SAGE Publications, Thousand Oaks, CA, 1990.

[28] Marshall, B., Cardon, P., Poddar, A., and Fontenot, R., "Does sample size matter in qualitative research?: A review of qualitative interviews in IS research", Journal of Computer Information Systems, 54(1), 2013, pp. 11-22.

[29] Germonprez, M., Kendall, J.E., Kendall, K.E., Mathiassen, L., Young, B., and Warner, B., "A theory of responsive design: A field study of corporate engagement with open source communities", Information Systems Research, 28(1), 2016, pp. 64-83.

[30] Becker, M., "Understanding Users' Health Information Privacy Concerns for Health Wearables", in Proceedings, 51st Hawaii International Conference on System Sciences (HICSS), Big Island, USA. 2018.

[31] Boyatzis, R.E., Transforming qualitative information: Thematic analysis and code development, SAGE Publications, Thousand Oaks, CA, 1998.

[32] Feldman, M.S., "Organizational Routines as a Source of Continuous Change", Organization Science, 11(6), 2000, pp. 611-629.

[33] Alter, S., "Work system theory: Overview of core concepts, extensions, and challenges for the future", Journal of the Association for Information Systems, 14(2), 2013, pp. 72-121.

[34] Laumer, S., Maier, C., Eckhardt, A., and Weitzel, T., "Work routines as an object of resistance during information systems implementations: Theoretical foundation and empirical evidence", European Journal of Information Systems, 25(4), 2017, pp. 317-343.

[35] Bala, H. and Venkatesh, V., "Changes in Employees' Job Characteristics During an Enterprise System Implementation: A Latent Growth Modeling Perspective", MIS Quarterly, 37(4), 2013, pp. 1113-1140.

[36] Malhotra, Y. and Galletta, D., "A multidimensional commitment model of volitional systems adoption and usage behavior", Journal of Management Information Systems, 22(1), 2005, pp. 117-151.

[37] Wang, Y., Meister, D.B., and Gray, P.H., "Social influence and knowledge management systems use: Evidence from panel data", MIS Quarterly, 37(1), 2013, pp. 299-313.

[38] Ajzen, I., "The theory of planned behavior", Organizational Behavior and Human Decision Processes, 50(2), 1991, pp. 179-211.

[39] Venkatesh, V., Morris, M.G., Davis, G.B., and Davis, F.D., "User acceptance of information technology: Toward a unified view", MIS Quarterly, 27(3), 2003, pp. 425-478.

[40] Bhattacherjee, A., Davis, C.J., Connolly, A.J., and Hikmet, N., "User response to mandatory IT use: A coping theory perspective", European Journal of Information Systems, 2018, pp. 1-20.

[41] Thompson, M., "People, practice, and technology: Restoring Giddens' broader philosophy to the study of information systems", Information and Organization, 22(3), 2012, pp. 188-207.

[42] Stein, M.-K., Newell, S., Wagner, E.L., and Galliers, R.D., "Coping with Information Technology: Mixed Emotions, Vacillation, and Nonconforming Use Patterns", MIS Quarterly, 39(2), 2015, pp. 367-392.

[43] Lapointe, L. and Rivard, S., "A multilevel model of resistance to information technology implementation", MIS Quarterly, 29(3), 2005, pp. 461-491.

[44] Jasperson, J.S., Carter, P.E., and Zmud, R.W., "A comprehensive conceptualization of post-adoptive behaviors associated with information technology enabled work systems", MIS Quarterly, 29(3), 2005, pp. 525-557. 